\title{
Laryngeal Cartilage
}

National Cancer Institute

\section{Source}

National Cancer Institute. Laryngeal Cartilage. NCI Thesaurus. Code C12281.

The nine cartilages that comprise the skeleton of the larynx, including the single thyroid, epiglottic, and cricoid cartilages, and the paired arytenoid, corniculate, and cuneiform cartilages. 